Research Article

\title{
SERS Platform Based on Bimetallic Au-Ag Nanowires- Decorated Filter Paper for Rapid Detection of miR-196a in Lung Cancer Patients Serum
}

\author{
Ji Xia, ${ }^{1,2,3,4}$ Yifan Liu, ${ }^{1,2,3,4}$ Menglin Ran, ${ }^{1,2,3,4}$ Dan Lu, ${ }^{2,3,4}$ Xiaowei Cao $\mathbb{D}^{1,2,3,4,5,6}$ \\ and Youwei Wang iD 1 \\ ${ }^{1}$ Department of Neurosurgery, Affiliated Hospital of Yangzhou University, Yangzhou 225001, China \\ ${ }^{2}$ Institute of Translational Medicine, Medical College, Yangzhou University, Yangzhou, China \\ ${ }^{3}$ Department of Obstetrics and Gynecology, College of Clinical Medicine, Yangzhou University, Yangzhou, China \\ ${ }^{4}$ The First Clinical College, Dalian Medical University, Dalian, China \\ ${ }^{5}$ Jiangsu Key Laboratory of Integrated Traditional Chinese and Western Medicine for Prevention and \\ Treatment of Senile Diseases, Yangzhou University, Yangzhou, China \\ ${ }^{6}$ Jiangsu Co-Innovation Center for Prevention and Control of Important Animal Infectious Diseases and Zoonoses, \\ College of Veterinary Medicine, Yangzhou University, Yangzhou 225009, China
}

Correspondence should be addressed to Xiaowei Cao; cxw19861121@163.com and Youwei Wang; wyw0520@126.com

Received 20 June 2020; Accepted 20 August 2020; Published 22 October 2020

Academic Editor: Gulaim A. Seisenbaeva

Copyright ( 2020 Ji Xia et al. This is an open access article distributed under the Creative Commons Attribution License, which permits unrestricted use, distribution, and reproduction in any medium, provided the original work is properly cited.

\begin{abstract}
Detecting microRNA (miRNA) biomarkers expression is of great significance for the diagnosis and treatment of lung cancer. Surface-enhanced Raman scattering (SERS) has achieved microRNA sensing for the diagnosis of primary liver cancers. In this work, we developed a SERS technology for the rapid detection of lung cancers-related miRNA (miR-196a) using bimetallic Au-Ag nanowire (AgNW@AuNPs) substrates coupled with the target hairpin DNA. The finite-difference time-domain simulation proved that a large number of "hot spots" were generated between the AgNW and AuNPs, which resulted in a huge enhancement of the signal of Raman reporters. Filter paper treated by hexadecenyl succinic anhydride hydrophobic and modified with AgNWs@AuNPs was used as capturing substrate. The detection limits of miR-196a in PBS and serum were as low as 96.58 aM and $130 \mathrm{aM}$, respectively. Studies on nonspecific sequence and single-base mismatch of miRNA demonstrated that SERS-based platform was highly selective, excellent uniform, and reproducible. Finally, the platform was used to show that the miR-196a expression in the serum of lung cancer patients was much higher than that in healthy people. The detection results indicated that the SERS platform had potential applications in cancer diagnosis and might be a viable alternative to the conventional miRNA detection method, the real-time polymerase chain reaction (RT-PCR) technology.
\end{abstract}

\section{Introduction}

MicroRNAs (miRNAs) are a class of small, highly conserved, noncoding RNAs that play critical regulatory roles in a range of biological processes, including cell proliferation, differentiation, apoptosis, and immunoregulation [1, 2]. The abnormal expression of miRNAs is related to many pathological processes, such as metabolic disorders and immune system dysfunction. Some miRNAs actually function as oncogenes or tumor suppressors in cancers [3]. Many studies have indicated that the expression of miR-196a is significantly upregulated in the serum of different tumor patients and revealed that miR-196a is involved in the proliferation, migration, and invasion of a number of cancer cells (gastric cancer, hepatocellular carcinoma, cervical cancer, non-Hodgkin lymphoma, and non-small cell lung cancer) [4-7]. Therefore, the detection of miR-196a expression is vital for the diagnosis of tumors. Traditional detection methods for miRNAs, such as Northern hybridization, in situ hybridization, real-time quantitative PCR, 
and microarray technology, are complex and time-consuming and exhibit low sensitivities, and, thus, they have difficulty meeting the needs of cell sample detection. Therefore, it has become an urgent problem to explore a miRNA detection technique using a simple operation with high sensitivity and strong specificity.

Surface-enhanced Raman scattering (SERS) is a powerful spectroscopy technique for the quantitative detection of molecules located closely or bounding to the plasmonic metal surface $[8,9]$. SERS can be used to obtain abundant molecular and structural information to identify biological samples with vibration fingerprints [10]. The detection sensitivity of SERS is similar to fluorescence techniques by as much as 6 to 14 orders of magnitudes higher than conventional Raman spectroscopy $[11,12]$. Moreover, SERS also has many significant advantages over other technologies, such as its narrow spectral band and weak Raman signal of water, in addition to being nondestructive [13]. Therefore, it is suitable for the study of biomolecules in water-containing systems. SERS has shown great research value and the application potential in the field of miRNA detection. Zhang et al. [14] showed that the SERS frequency shift method combined with microcontact printing is a genuine candidate among others in development to offer a cheaper, less cumbersome, and more accurate approach to multiplex assaying of serum microRNAs for the early detection and discrimination of primary liver cancers. Zhu et al. [15] developed an RNA site embedding the DNA-rN1-DNA-mediated SERS frequency shift sensing platform for the first time for the ctDNA assay, which exhibited subfemtomolar-level detection sensitivity. Guven et al. [16] constructed SERS probes using Raman 5,5'-dithiobis (2-nitrobenzoic acid) (DTNB) and miRNA-21-modified Au nanorods and fixed the DNA chains on the surfaces of the Au substrates to form capture probes, which could be paired with SERS probes to form Sandwich detection structures. With the enhanced performance of the SERS probes and the SERS signal of DTNB, the detection limit of the highly sensitive miR-21 was $0.85 \mathrm{nM}$. Pang et al. [17] combined SERS with ELISA to detect let-7b in HeLa, MCF-7, and A549 cells with high sensitivity. They connected let-7b with $\mathrm{Cy} 3$ that was modified at the $3^{\prime}$ end to the surface of $\mathrm{Fe}_{3} \mathrm{O}_{4} @ \mathrm{Ag}$. With the complementary pairing of DNA and let-7b and the restriction endonuclease acting on the complementary double-strand, the "hot spots" between the nanoparticles disappeared and the SERS signal intensity weakened. The let- $7 \mathrm{~b}$ detection could be realized based on the change of the SERS signal, and the detection limit was up to $0.3 \mathrm{fM}$.

SERS enhancement effects mainly result from two enhancement mechanisms, a strong long-range electromagnetic (EM) enhancement and a weaker short-range chemical enhancement. The EM enhancement arises from localized surface plasmon oscillations on the nanostructured noble metallic substrate under laser excitation with amplified vibrational and rotational Raman modes of the reporter compound positioned within close proximity [18]. To obtain reliable SERS measurements, the substrate materials are vital. Hybrid metal-metal nanostructures have attracted widespread attention. They integrate two different functions into a single entity with unprecedented performance, which is critical for the further development of nanostructure designs for chemical and biological sensing applications $[19,20]$. Among the many metal-metal hybrid nanostructures, $\mathrm{Au}-\mathrm{Ag}$ composites with different structures have been widely used in various fields, especially in the field of SERS sensing. Ag and $\mathrm{Au}$ nanoparticles have unique absorption peaks in the visible and near-infrared regions, and their optical properties are adjustable in shape and size $[21,22]$. Nanoparticles and nanowires are two important materials of SERS nanostructures because of their simple preparation processes. However, due to the mismatch of light dispersion, long uniform metal nanowires cannot be directly coupled with light. When $\mathrm{Au}$ nanoparticles (AuNPs) and Ag nanowires (AgNWs) are close to each other, they can be coupled to form hot spots and enhance the Raman signals. AuNPs can act like nanoantennas and light can propagate the plasma coupling between the AuNPs and AgNWs [23]. When the probe molecule is located between the particle and the line, the field enhancement can enhance the Raman scattering signal substantially. Thus, $\mathrm{Au}-\mathrm{Ag}$ nanowires (AgNW@AuNPs) are suitable for the preparation of SERS-reinforced substrates. A variety of methods (e.g., template method, self-assembly, nanosphere lithography, and electrochemical deposition) had been used to assemble nanoparticles on solid substrates to obtain greater sensitivity and signal enhancement.

Recently, by the advantages of low-cost, flexibility, portability, and biodegradation, the preparation of paperbased SERS substrates has been widely interested [24-26]. Compared with the traditional solid supports which had shown high reproducibility and sensitivity with suitable nanostructures, the natural fold and fiber structure of paper were beneficial to the uniform distribution of metal nanoparticles and the formation of SERS hot spots [27]. However, the hydrophilicity of filter paper promoted the uniform distribution of metal nanomaterials and also made the sample solution easy to be adsorbed into the filter paper as well as random distribution on a large area, which made the SERS signal of paper-based substrate weaken, with poor sensitivity and reproducibility [28]. There were many methods to process filter paper, including inkjet printing [29], solution dipping [30], physical vapor deposition [31], but these methods are complex and expensive. In this work, a new synthesis technology of hydrophobic filter paper was proposed. The filter paper was treated with hexadecyl succinic anhydride (HDSA) and the hydroxyl groups on the surface of the filter paper were replaced by long-chain carbene groups of HDSA to obtain hydrophobic filter paper. The homogeneity and repeatability of the matrix were improved by preventing analytes and nanoparticles in HDSAtreated filter paper from being absorbed quickly and reacting with the matrix sufficiently.

Herein, a hairpin DNA-based SERS platform was constructed for the detection of miR-196a. The DNA 
hairpin sequence was designed complementarily to miR196a and functionalized with the thiol group at the $5^{\prime}$-end, and 5-carboxyfluorescein (5-FAM) was used as Raman reporter which was labeled on the $3^{\prime}$-end of DNA. The analysis based on the SERS platform is schematically illustrated in Figure 1. The AgNW@AuNPs were prepared by the electrostatic interaction method and subsequently assembled onto the surfaces of filter paper treated by hexadecenyl succinic anhydride hydrophobic (HDSA). The optimal assembly and hybridization time as well as the sensitivity, specificity, uniformity, and reproducibility of the SERS platform were investigated systematically. Based on the hairpin DNA-coupled AgNW@AuNPs substrates, SERS was applied to analyze the miR-196a simultaneously in phosphate-buffered saline (PBS) and the serum. Finally, the miR-196a expression in serum of lung cancer patients and healthy people was detected by SERS. The feasibility of the SERS was proved by PCR experiments. SERS platform may be used for detecting other cancer biomarkers and it has shown potential for screening multiple cancers.

\section{Materials and Methods}

2.1. Materials. Ultra-pure water produced by Milli-Q (Millipore, USA, resistivity $>18 \mathrm{M}$ ) was employed in the experiments. Chloroauric acid tetrahydrate $\left(\mathrm{HAuCl}_{4} \cdot 4 \mathrm{H}_{2} \mathrm{O}\right), \mathrm{Ag}$ nitrate $\left(\mathrm{AgNO}_{3}\right.$ ), polyvinylpyrrolidone (PVP, $\left.\mathrm{K} 30\right), \mathrm{NaBH}_{4}$, phosphate $\left(\mathrm{NaH}_{2} \mathrm{PO}_{4} \cdot 2 \mathrm{H}_{2} \mathrm{O}\right.$ and $\left.\mathrm{Na}_{2} \mathrm{HPO}_{4} \cdot 12 \mathrm{H}_{2} \mathrm{O}\right)$, ethanol $\left(\mathrm{CH}_{3} \mathrm{CH}_{2} \mathrm{OH}\right)$, hexadecenyl succinic anhydride (HDSA), filter paper, and ethylene glycol (anhydrous, 99.8\%) were bought from Yangzhou Younuo Chemicals Co. Ltd. (China). All the chemicals were utilized in the experiments without any further purification processes. Blood samples were collected from lung cancer patients and healthy people from the clinical medical college of Yangzhou University in Jiangsu Province. The blood samples were divided into two groups: 30 healthy people and 30 lung cancer patients. The age and sample size of the two groups are shown in Table 1. All written consent and moral permission have been obtained. Blood samples were centrifuged at $3000 \mathrm{rpm}$ at $4^{\circ} \mathrm{C}$ for 15 minutes, and the supernatant obtained was preserved at $-80^{\circ} \mathrm{C}$. The miR-196a, hairpin DNA, miR-10a, single-base mismatch (relative to miR-196a), and PCR kits for miR-196a were purchased from Sangon Biotech (Shanghai) Co., Ltd., as shown in Table 2. All the glassware was cleaned with aqua regia and ultra-pure water.

2.2. Preparation of Ag Nanowires. AgNWs were prepared following a modified method of that presented by Li et al. [20] During the preparation of AgNWs, $10 \mathrm{~mL}$ of ethylene glycol (EG) was injected into the beaker at $160^{\circ} \mathrm{C}$ and stirred for 1 hour. Next, $\mathrm{AgNO}_{3}(4 \mathrm{~mL}, 0.3 \mathrm{mM})$ and PVP $(5 \mathrm{~mL}, 0.2 \mathrm{mM})$ in EG were quickly added to the beaker. The reaction continued until the color of the mixture changed to light gray. Afterwards, the mixture was cleaned by acetone two times and absolute ethanol three times. To give the negative charges on surfaces of AgNWs, PVP was employed as a soft template and surface stabilizer in the preparation of AgNWs [32].
2.3. Preparation of AgNW@AuNPs. First, $\mathrm{NaBH}_{4}(20 \mathrm{~mL}$, $0.15 \mathrm{mM})$ was dropped to $\mathrm{HAuCl}_{4}(50 \mu \mathrm{L}, 24.3 \mathrm{mM})$ and stirred vigorously to prepare the AuNPs. After 40 minutes, the AuNPs mixture was kept in a refrigerator of $4^{\circ} \mathrm{C}$. Next, $50 \mu \mathrm{L}$ of the AuNP mixture charged with positively was added in $1 \mathrm{~mL}$ of the AgNW ethanol solution charged with negatively at room temperature with strong stirring. After 4 hours, AuNPs were set on the surfaces of the AgNWs. Finally, the prepared samples were cleaned three times with ethanol to remove the unmodified AuNPs on AgNWs.

2.4. Synthesis of Capturing Substrates. To prepare a sensitive and uniform filter paper substrate, 10\% hexanol HDSA solution as a hydrophobic agent was sprayed on the surface of the filter paper and placed in an oven at $100^{\circ} \mathrm{C}$ for 10 minutes. Then, the filter paper was cut into $90 \mathrm{~mm} \times 90 \mathrm{~mm}$ size and immersed directly in AgNW@AuNPs solution for 24 hours. Finally, the filter paper was dried naturally at room temperature to obtain the substrate. The hairpin DNA immobilization on the AgNW@AuNP substrates is shown in Figure 1. The hairpin DNA-5FAM mixture was incubated at $95^{\circ} \mathrm{C}$ for 5 minutes and then, they reacted in an ice-water continually for 20 minutes to refine the hairpin structure. In the closed state, 5-FAM was the closest to the surface of the substrate $(<1 \mathrm{~nm})$, which conduced to the stronger SERS signal intensity [33]. The purified hairpin DNA in a PBS solution was dropped onto the SERS substrate in the thermotank with a temperature of $25^{\circ} \mathrm{C}$ as well as a humidity of $\sim 80 \%$. After 3 hours, the hairpin DNA-modified substrate was put in PBS buffer for about 30 minutes. The resultant hairpin DNA-modified substrate was cleaned by PBS buffer. Also, the hairpin DNA-modified SERS platform was subsequently blocked by a $1 \%$ BSA solution to eliminate the nonspecific binding. After washing with PBS, the SERS platforms were obtained.

2.5. Detection of miR-196a. The as-prepared SERS platforms were soaked in a sterile 24 -well plate and incubated at $37^{\circ} \mathrm{C}$ as well as $\sim 80 \%$ humidity for 2 hours to ensure the hybridization of the hairpin DNA and target miRNA. After washing by water, the wet substrate was used to obtain the SERS spectra. Figure 1 shows the SERS detection procedure for the lung cancer-related miR-196a in the cell lysate. After the hybridization of the complementary targets and hairpin DNA, the relative rigid linear DNA-RNA duplexes were obtained by the opening of the configurations of hairpin DNA. Under these circumstances, the Raman labels were separated away from the substrates, which led to a significant decrease in the SERS intensity.

2.6. SERS Measurement. Nile Blue A (NBA) and 5-carboxyfluorescein (5-FAM) were used as Raman labels. The inVia-Reflex Renishaw Raman system at the range of $600-1800 \mathrm{~cm}^{-1}$, which could obtain an excitation wavelength of $785 \mathrm{~nm}$ from a He-Ne laser in line-focus mode with a laser power of $5 \mathrm{~mW}$, was used to collect SERS spectra. The $50 \times$ long working distance objective was used to focus on the 


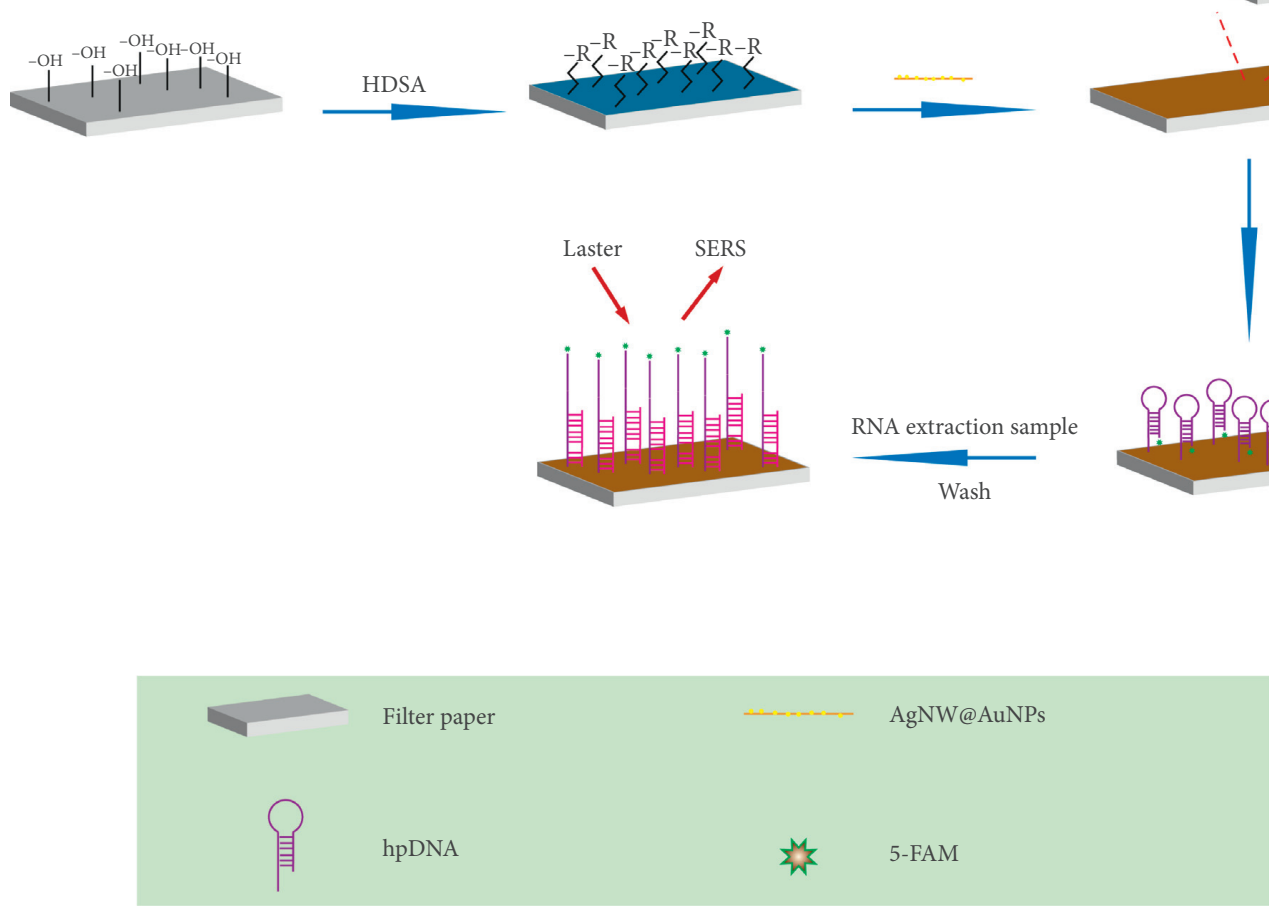

FIGURE 1: Scheme of the hairpin DNA-functionalized SERS platform for rapid detection of miR-196a expression in serum of lung cancer patients.

TABLE 1: Demographics of volunteers.

\begin{tabular}{lcc}
\hline Groups & Healthy people & Lung cancer patients \\
\hline Mean age (years) & 32 & 57 \\
Sample & 30 & 30 \\
\hline
\end{tabular}

TABLE 2: Sequences of DNA hairpin sequence, target miRNAs, single-base mismatch, and noncomplementary miRNAs.

\begin{tabular}{lc}
\hline Name & \multicolumn{1}{c}{ Sequence } \\
\hline Hairpin DNA & 5'-SH-CCCGAAYCACAGTGAAACTACTAAGAGTAAGAGACCTTTAA-5FAM-3' $^{\prime}$ \\
miR-196a & $5^{\prime}$-UAGUAGUUUCACUGUGAUUCGGG-3' \\
miR-10a & $5^{\prime}$-UACCCUGUAGAUCCGAAUUUGUG-3' \\
Single-base mismatch (relative to miR-196a) & $5^{\prime}$-UAGUAGUUUCACUGAGAUUCGGG -3' \\
\hline
\end{tabular}

surface of the sample to a spot by corresponding laser with approximately $2 \mu \mathrm{m}$ diameter. The spectra were collected in continuous mode with an exposure time of $10 \mathrm{~s}$ and accumulated twice using a $1200 \mathrm{~mm}^{-1}$ grating. Ten randomly locations were selected on the SERS platform for each sample to obtain SERS spectra and the averaged SERS spectrum was obtained according to the ten SERS scans.

2.7. Characterization. Tecnai 12 transmission electron microscope which was operated at an accelerating voltage of $60 \mathrm{kV}$ (Philips) was used to take transmission electron microscopy (TEM) images. Scanning electron microscopy (SEM) images were acquired using an S-4800 II field-emission scanning electron microscope (FESEM) operating at $3.0 \mathrm{kV}$ (Hitachi). The high-resolution TEM images (HRTEM) and selected area electron diffraction (SAED) images were captured by Tecnai G2 F30 S-Twin TEM at $200 \mathrm{kV}$ (FEI) to observe the crystalline structure of the particles.

\section{Results and Discussion}

3.1. Characterization of Ag Nanowires and AgNW@AuNPs. The SEM and TEM were employed to observe the morphology and structure of AgNWs and AgNW@AuNPs. Figures 2(a) and 2(b) indicate that the AgNWs were well dispersed and the surfaces were quite smooth. As illustrated in Figure 2(g), the peaks of Ag shown in the energy-dispersive X-ray spectroscopy (EDX) spectrum of the AgNWs 


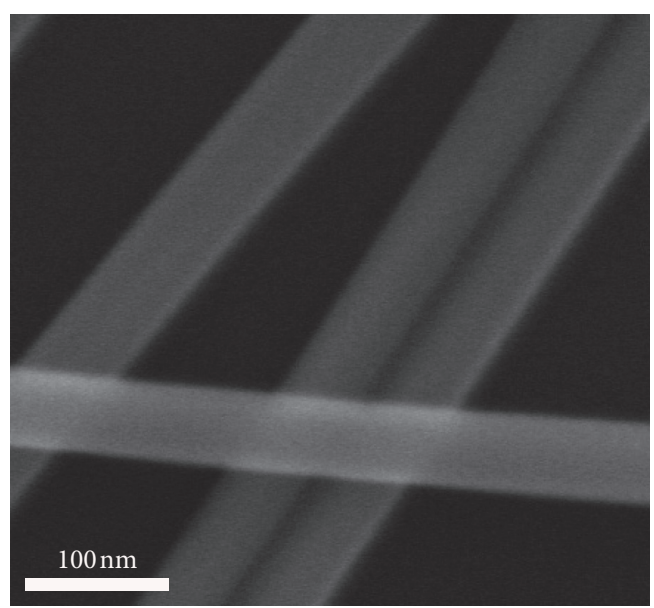

(a)

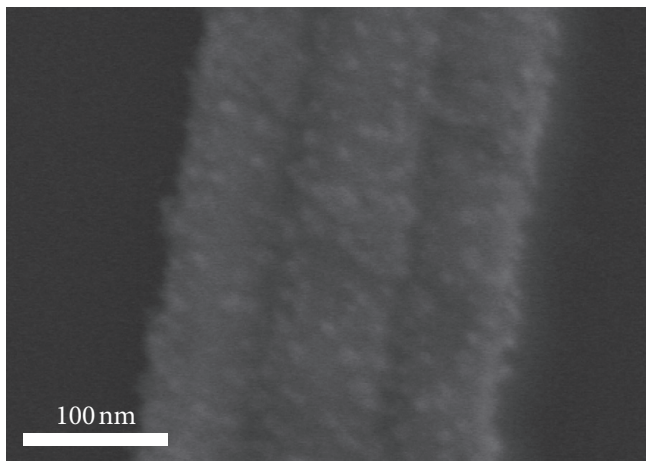

(c)

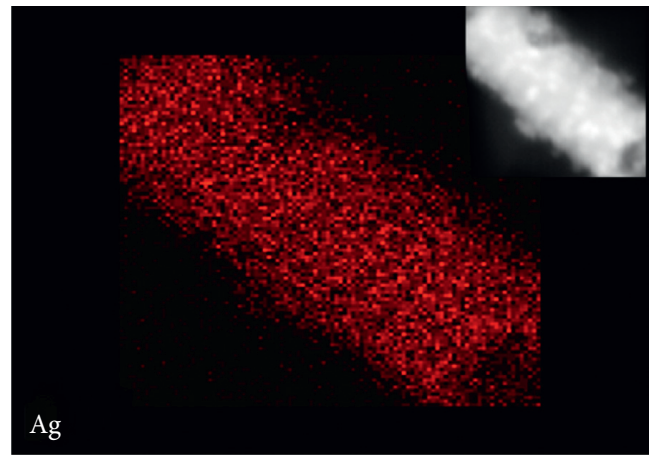

(e)

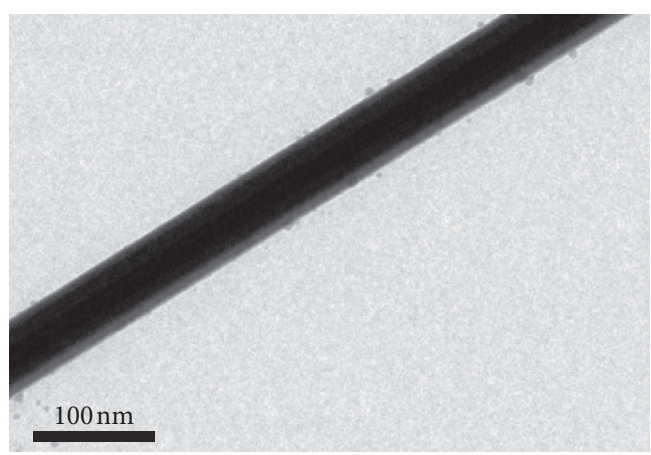

(b)

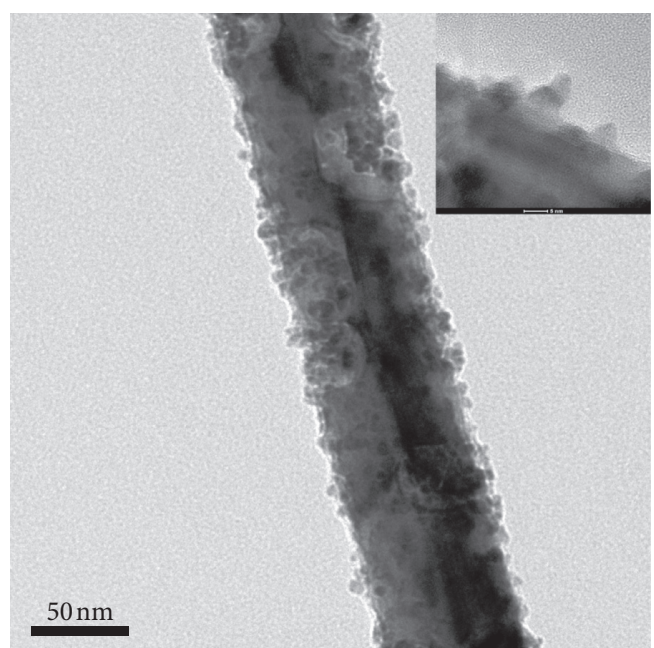

(d)

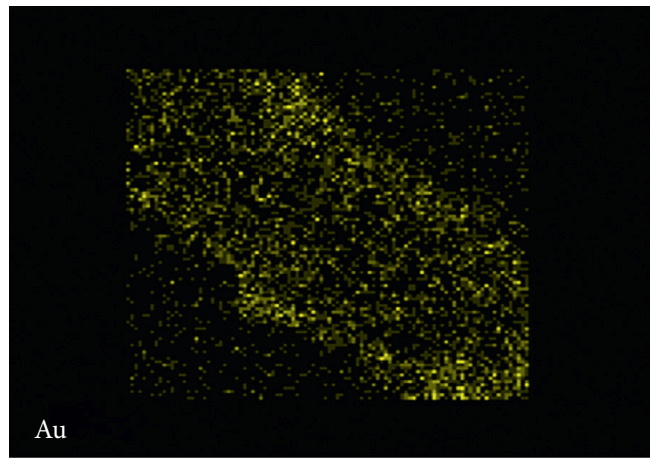

(f)

FIgURE 2: Continued. 


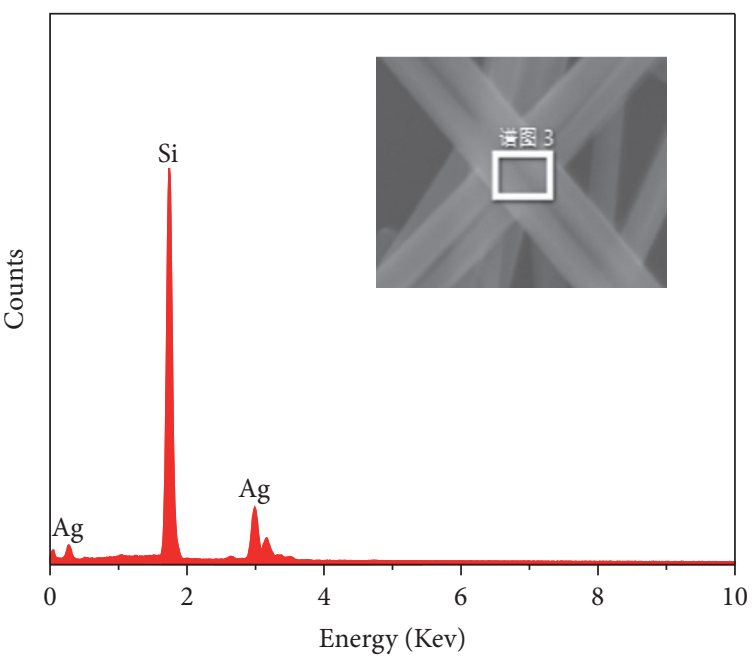

(g)

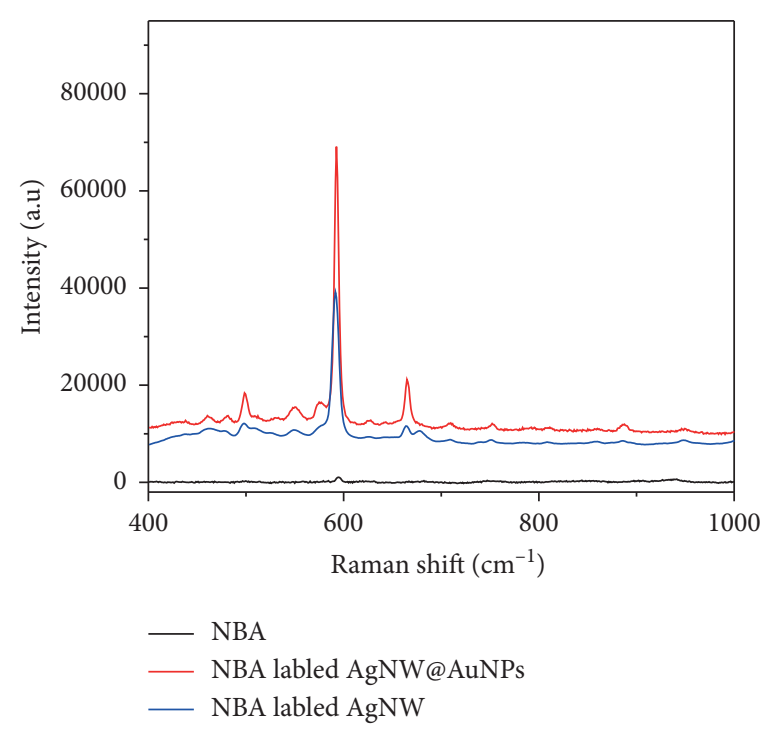

(i)

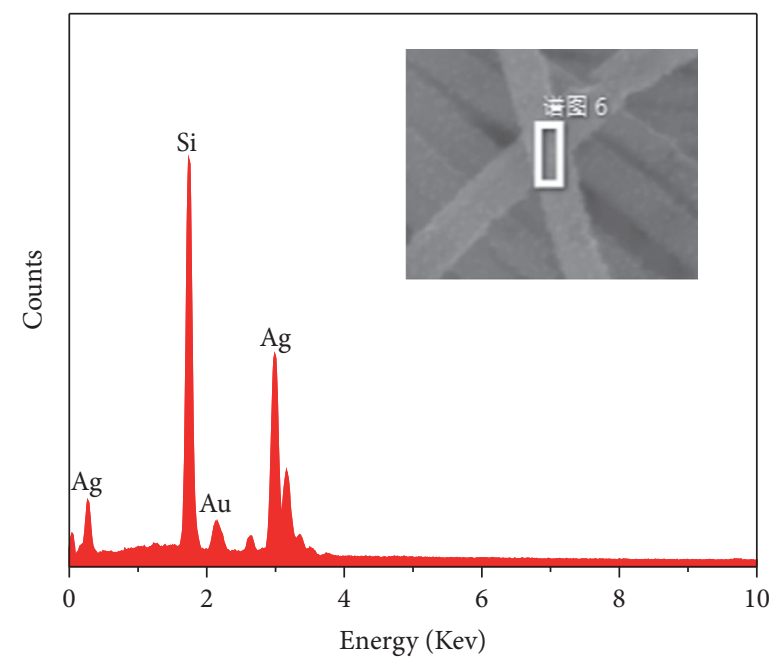

(h)

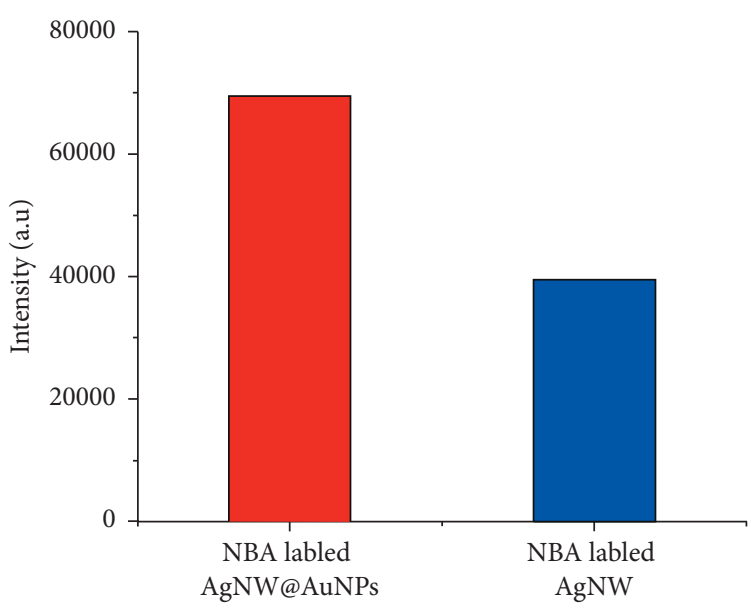

(j)

Figure 2: The images of (a) SEM and (b) TEM of the AgNW. (c) SEM images and (d) TEM images of the AgNW@AuNPs. EDX mapping of (e) Ag element and (f) Au element in AgNW@AuNPs. EDX spectrum of (g) AgNW and (h) AgNW@AuNPs. (i) SERS spectra of NBA, NBAlabeled AgNW, and NBA-labeled AgNW@AuNPs. (j) The intensity of the peaks at $592 \mathrm{~cm}^{-1}$ corresponding to the SERS spectra in (I).

indicated that the AgNWs were highly pure and the Si peak was due to the silicon wafer. The AgNW@AuNPs were prepared by setting the AuNPs charged with positive onto the surfaces of the Ag nanowires charged with negative via electrostatic interactions. The typical TEM and SEM images of the AgNW@AuNPs are shown in Figures 2(c) and 2(d). Compared to the smooth surfaces of the Ag nanowires, the surfaces of the AgNW@AuNPs became rough because of the adsorption of the AuNPs which had a diameter of $7.3 \mathrm{~nm}$. It was clear that the aggregation degree of gold nanoparticles on nanowires was different. As shown in Figures 2(e) and 2(f), EDS elemental mappings were taken to further investigate the elemental distribution of the AgNW@AuNPs. The homogeneous distributions of $\mathrm{Au}$ and $\mathrm{Ag}$ indicated the AuNPs were uniformly assembled on the surfaces of the
AgNWs. Also, the result of energy-dispersive X-ray spectroscopy (EDX) proved the composition of AgNW@AuNPs was mainly Ag and a small amount of Au (Figure 2(h)).

It was displayed in Figure 2(i) that the Raman spectra of the NBA, NBA-labeled AgNWs, and NBA-labeled AgNW@ AuNPs were employed to explore the SERS effect of the AgNW@AuNPs. The aromatic ring vibrations resulted in the characteristic peak of NBA at $592 \mathrm{~cm}^{-1}$ [34]. Compared to the slight variation of the SERS signal of the $0.1 \mathrm{M} \mathrm{NBA}$, the SERS signal of the NBA-labeled AgNW was lower than that of the NBA-labeled AgNW@AuNPs, which indicated that the AgNW@AuNPs could enhance the Raman signal intensity better. Figure 2(j) shows that the SERS intensity at $592 \mathrm{~cm}^{-1}$ of the NBA-labeled AgNW@AuNPs was twice as much as that of the NBA-labeled AgNW. The SERS 
enhancement effect of the AgNW@AuNPs was verified by enhancement factor (EF), $\mathrm{EF}=\left(\mathrm{I}_{\text {SERS }} / \mathrm{C}_{\mathrm{SERS}}\right) /\left(\mathrm{I}_{\mathrm{RS}} / \mathrm{C}_{\mathrm{RS}}\right)$, where I IERS was the SERS intensity obtained for the AgNW@ AuNP colloidal dispersion at a certain concentration $\left(\mathrm{C}_{\text {SERS }}\right)$ of the analyte, and $\mathrm{I}_{\mathrm{RS}}$ was the Raman intensity obtained under non-SERS conditions at an analyte concentration $\left(\mathrm{C}_{\mathrm{RS}}\right)$ [35]. In the course of the experimental, the AgNW@ AuNPs colloidal dispersion was added into the same concentration of NBA solution $\left(2 \times 10^{-6} \mathrm{M}\right)$ for 2 hours to gain a final concentration of $10^{-6} \mathrm{M}$. When $\mathrm{C}_{\text {SERS }}$ was $1 \times 10^{-6} \mathrm{M}$ and $C_{R S}$ was $0.1 \mathrm{M}$, a value of $\mathrm{EF}=5.03 \times 10^{6}$ was obtained.

3.2. FDTD Simulation of AgNW@AuNPs. To reveal the optical properties of the AgNW@AuNPs and confirm the formation of hotspots, distributions of the electromagnetic field were investigated by finite-difference time-domain (FDTD) simulations. The simulation region was set as a unit $800 \mathrm{~nm} \times 800 \mathrm{~nm} \times 800 \mathrm{~nm}$ in 3Ds. Also, boundary conditions were set as perfectly matched layers (PML) in all simulations. To improve computational accuracy, a refined mesh grid near the structure was set as $0.1 \mathrm{~nm}$ in a $3 \mathrm{D}$ dimension $130 \mathrm{~nm} \times 100 \mathrm{~nm} \times 100 \mathrm{~nm}$. Total-field scatteredfield (TFSF) linearly polarized light waves, which is polarized in line with AgNWs ( $X$-axis) with a wavelength range of $400-1000 \mathrm{~nm}$, were injected into the unit cell along the $Z$ direction. All geometric parameters for simulations were consistent with the average actual size of the as-prepared samples shown in TEM image (Figure 3(a)) and the substrate was not included. The diameter and length of AgNWs were $68.6 \mathrm{~nm}$ and $700 \mathrm{~nm}$, respectively, while the diameter of the decorated AuNPs arrayed on the AgNWs was $7.3 \mathrm{~nm}$ and the excitation wavelength was $785 \mathrm{~nm}$. Figure 3(b) shows the simulated local electric field distribution in the $X-Z$ plane. It could be seen from Figure 3(b) that the enhanced electric field was not evenly distributed on the whole surface of AgNW@AuNPs, which resulted in the fact that the coupling effect of surface plasmons made the gaps of the AgNPs and AgNWS appear as hot spots with high electric field intensity. The above results showed that AgNW@AuNPs had a strong Raman enhancement effect, which was conducive to collecting more biochemical spectral information.

3.3. Characterization of AgNW@AuNPs Substrate. The AgNW@AuNP substrate was fabricated by HDSA-hydrophobic surface-assembly method. The filter paper was treated by the hydrophobic HDSA and the long-chain carbene of HDSA was used to replace the hydroxyl group on the surface of the filter paper, which resulted in the change of filter paper properties so that the analytes and the substrate could react fully. Figure 4(a) shows an SEM image of the AgNW@AuNPs, which indicated that the nanowires aggregated slightly and were evenly distributed on the surface of the filter paper. SERS mapping experiment was taken out after the NBA adsorbed on the surface $\left(1 \times 10^{-5} \mathrm{M}\right)$ to research the surface homogeneity of the SERS signal. The scanning range was $70 \times 70 \mu \mathrm{m}^{2}$ and the length was $2 \mu \mathrm{m}$ with the laser power of $5 \mathrm{~mW}$. The characteristic peak at $592 \mathrm{~cm}^{-1}$ was plotted at each grid point on the substrate. As shown in Figure 4(b), the intensity of the $592 \mathrm{~cm}^{-1}$ characteristic peak at each grid point is shown in the color matching scheme from blue (minimum intensity) to green, yellow, orange, and red (maximum intensity). To further verify the uniformity of hydrophobic filter paper substrate, SERS mapping of ordinary filter paper substrate and hydrophobic filter paper substrate was compared (supporting information). According to the comparison between Figures 4(b) and $\mathrm{S} 1$, it was shown that although some polymers could still be found, hydrophobic filter paper substrate had a uniform SERS enhancement effect compared with ordinary filter paper substrate. The hydrophobic reagent changed the hydrophilic filter paper from hydrophilic to hydrophobic, which made the sample more evenly distributed on the hydrophobic filter paper matrix and improved the uniformity of the substrate.

\subsection{Characterization of Hairpin DNA-Functionalized SERS Platform}

3.4.1. Optimal Assembly Time of Hairpin DNA. The hairpin DNA assembly time determined the amount of hairpin DNA assembled on the nanowire surfaces. Figure 4(c) shows the SERS spectra of 5-FAM which were obtained from different times from 0 to 225 minutes. The characteristic peaks of the 5-FAM at $1334 \mathrm{~cm}^{-1}, 1640 \mathrm{~cm}^{-1}$, and $1184 \mathrm{~cm}^{-1}$ could be clearly identified $[36,37]$. The SERS intensity at $1334 \mathrm{~cm}^{-1}$ was set as a function of time in Figure 4(d), which showed that when the time was less than 90 minutes, the peaks at $1334 \mathrm{~cm}^{-1}$ increased almost linearly with time, after which the increase gradually slowed until it reached $195 \mathrm{~min}$. The peaks at $1334 \mathrm{~cm}^{-1}$ nearly reached a plateau at $195 \mathrm{~min}$, which indicated that the hairpin DNA had been fixed on the nanowire surfaces with the maximum quantity and achieved the dynamic balance of the hairpin DNA immobilization was achieved. Therefore, the best fixed time of the hairpin DNA was 195 minutes.

3.4.2. Optimal Hybridization Time. The hybridization time of the platform and targets relied on the time when the target miR-196a effectively hybridized with the hairpin DNA, and this time is important for achieving a good performance of the SERS platform [38]. The time-dependent SERS study was carried by using the SERS substrate with the optimal time as mentioned above. The SERS platform was put in miR-196a solution $(3 \mathrm{~mL}$, $100 \mathrm{pM}$ ) for further hybridization. The substrate was taken out and slowly cleaned by PBS buffer with SERS measurements immediately every $10 \mathrm{~min}$. The substrate was immersed in the hybridization mixture again and the above process was repeated. As shown in Figure 4(e), the relationship between the peak at $1334 \mathrm{~cm}^{-1}$ and time was established and plotted as blue triangles. The Raman intensity quenched quickly for times under 50 minutes. The peak at $1334 \mathrm{~cm}^{-1}$ declined slowly when time 


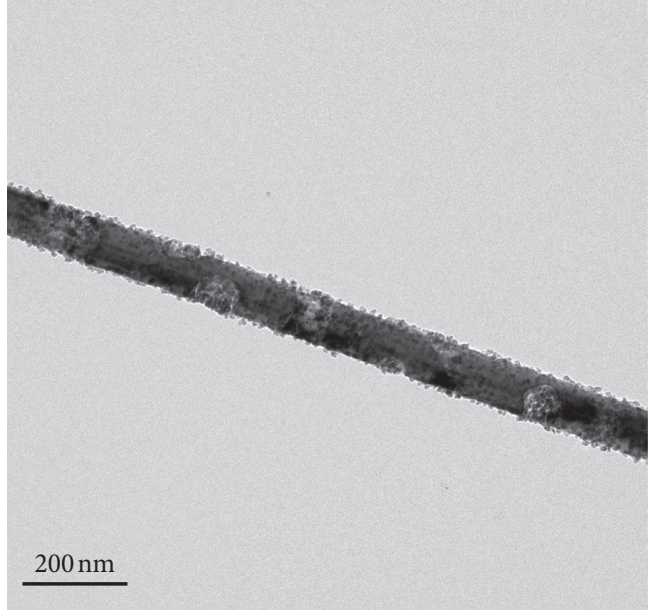

(a)

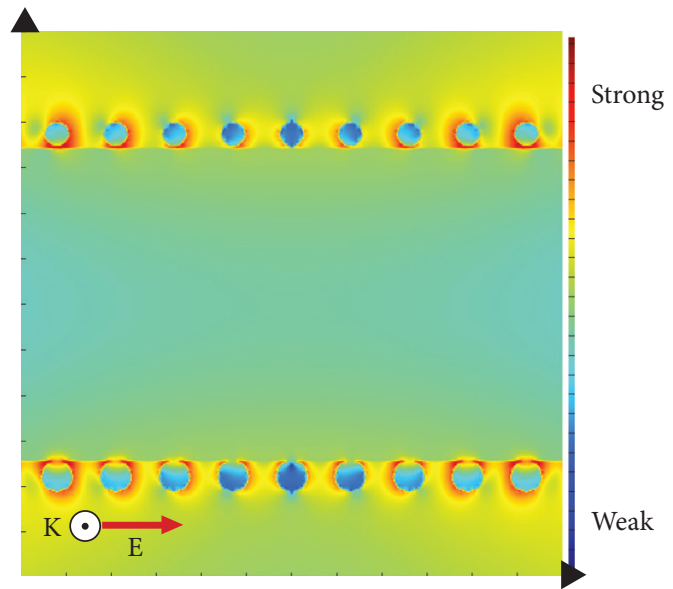

(b)

Figure 3: The electric field intensity distribution simulation of local electric field distribution for AgNW@AuNPs in the $X$ - $Z$ plane with $785 \mathrm{~nm}$ incident light. TEM images of AgNW@AuNPs (a) and its corresponding simulated electric field intensity distribution images (b).

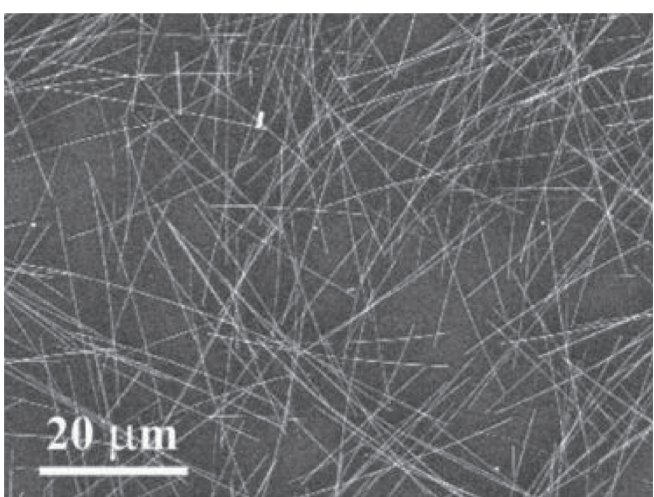

(a)

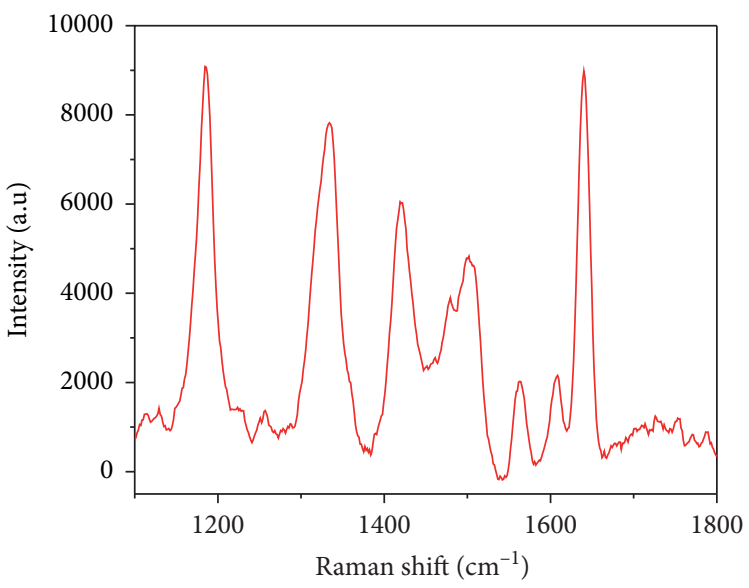

(c)

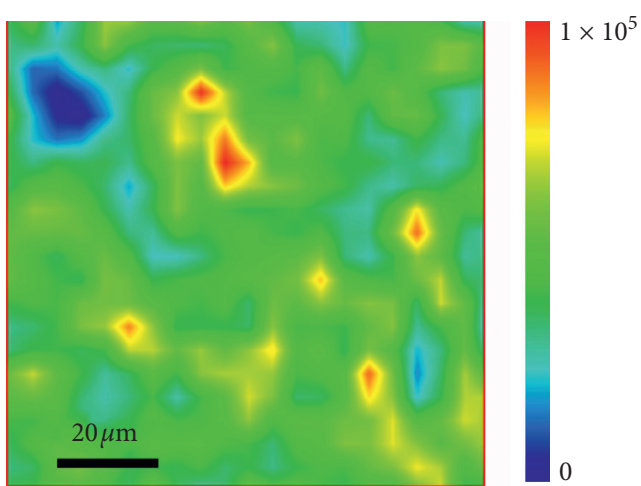

(b)

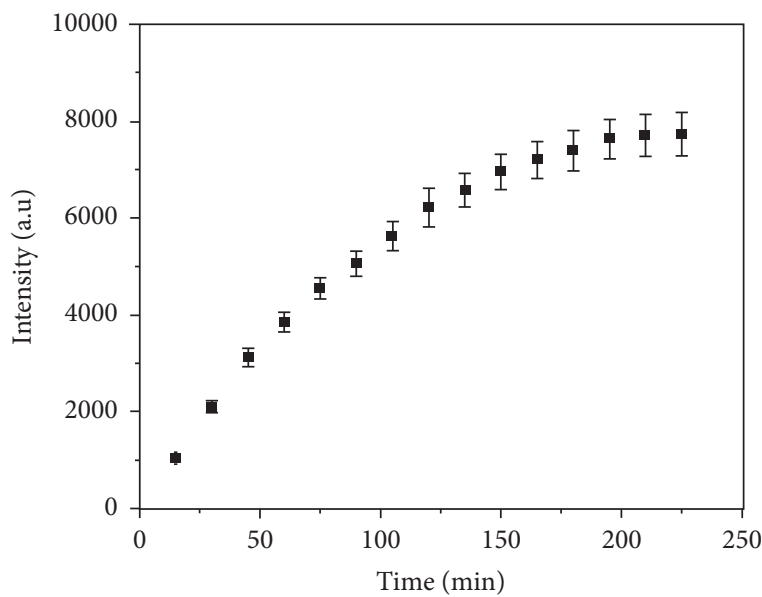

(d)

Figure 4: Continued. 

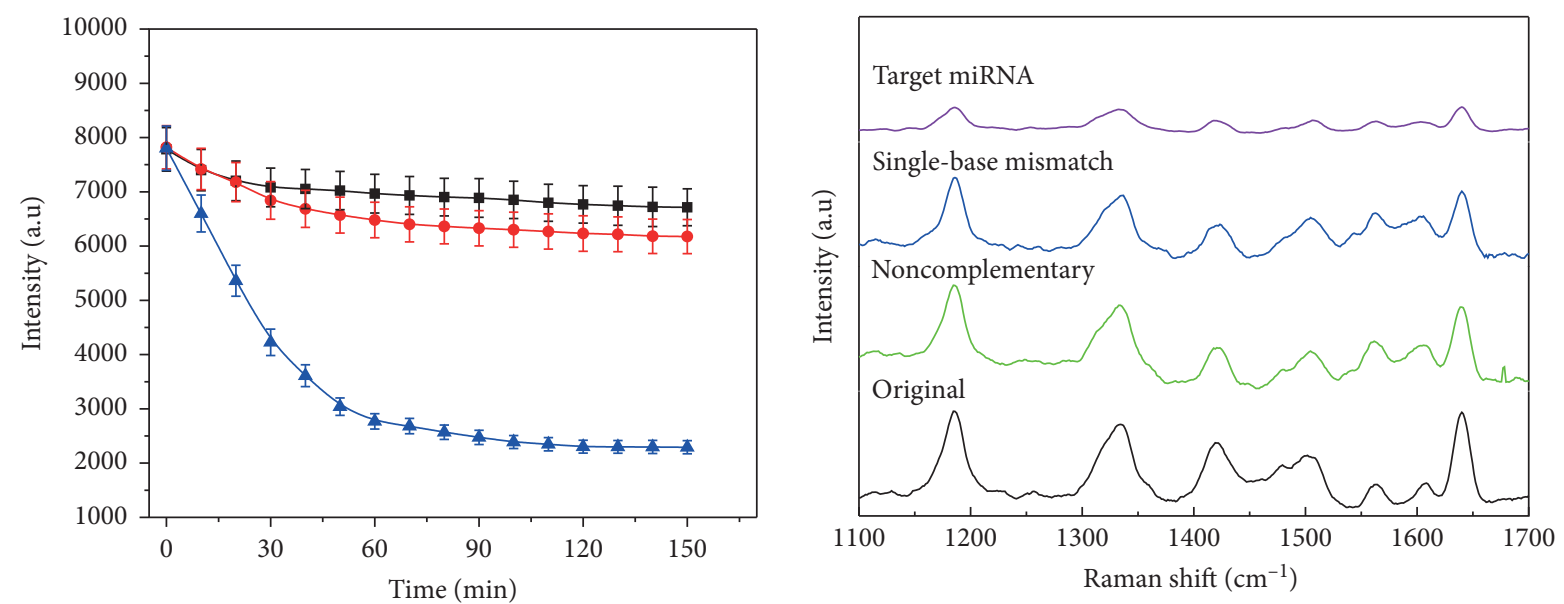

- Noncomplementry

$\rightarrow$ Single-base mismatch

$\simeq$ miRNA-196a

(e)
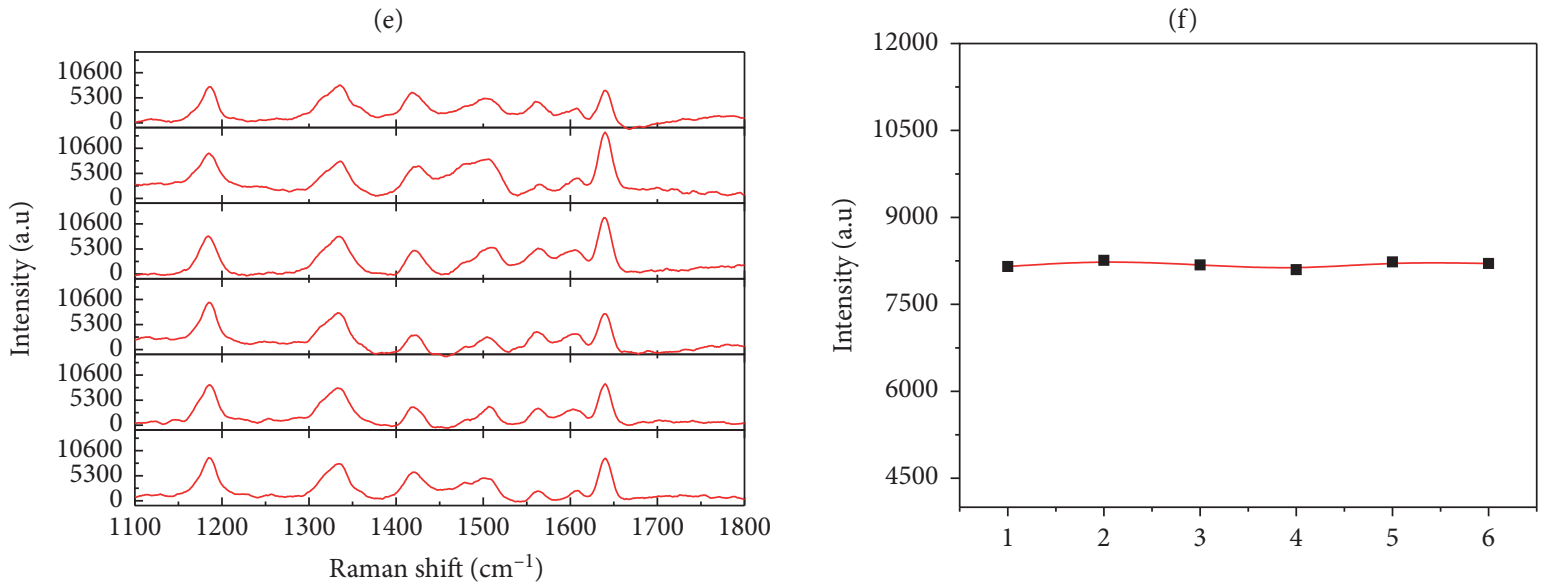

(g)

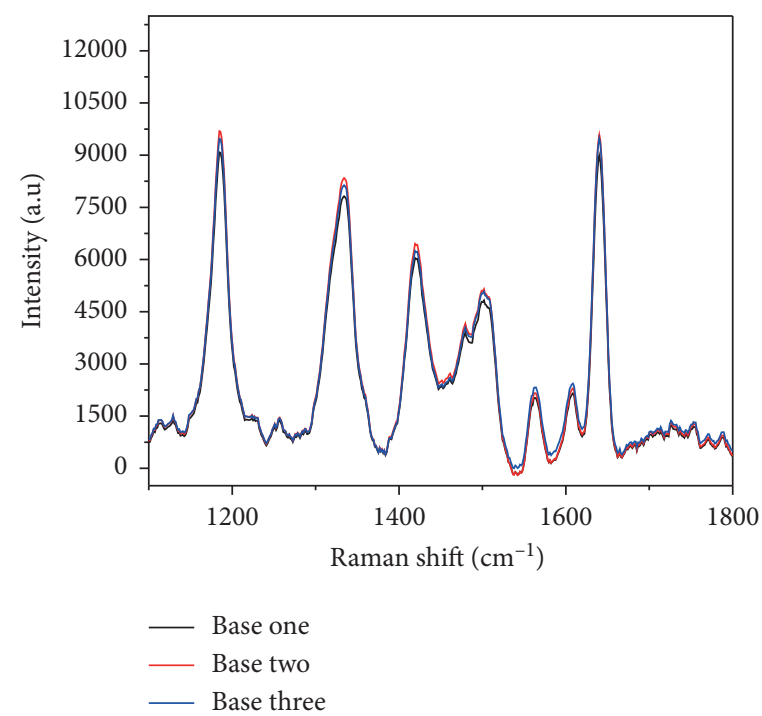

(h)

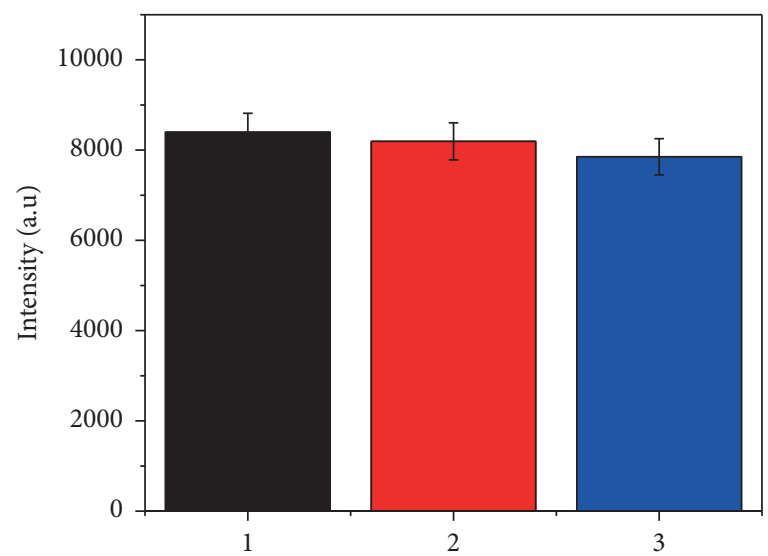

(i)

(j)

FIgUre 4: (a) SEM images of the AgNW@AuNPs substrate. (b) SERS mapping of NBA measured at $592 \mathrm{~cm}^{-1}$ using the AgNW@AuNPs substrate. (c) SERS spectrum of the AgNW@AuNPs substrate labeled DNA hairpin probe. (d) Assembly time-dependent SERS intensity at $1334 \mathrm{~cm}^{-1}$. (e) Time change of SERS intensity for the complementary miRNA (blue triangle), single-base mismatch miRNA (red circle), and noncomplementary miRNA (black square). (f) SERS spectra of SERS platform before (original) and after incubation with $100 \mathrm{pM}$ complementary, noncomplementary, and single-base mismatch miRNA. (g) SERS spectra at $1334 \mathrm{~cm}^{-1}$ from 6 random spots of the SERS platform after hybridization with $100 \mathrm{pM}$ miR-196a. (h) The line chart of the intensity at $1334 \mathrm{~cm}^{-1}$ corresponding to the SERS spectra in (g). (i) The reproducibility of the SERS platform. (j) The histogram of the intensity at $1334 \mathrm{~cm}^{-1}$ corresponding to the SERS spectra in (I). 
increased longer and finally reached relative stability when the time was more than 120 minutes. The decay of the peak at $1334 \mathrm{~cm}^{-1}$ before and after the SERS platform hybridization with the targeted miR-196a indicated that the hairpin DNA fixed on the substrate maintained perfect hybridization activity for miR-196a; meanwhile, the optimal hybridization time was 120 minutes to obtain the maximum and stable SERS signal change.

3.4.3. Specificity of SERS Platform. The specificity of the SERS platform was studied by detecting $100 \mathrm{pM}$ noncomplementary miRNA (miR-10a) and single-base mismatch miRNA (listed in Table 2). The relationship between $1334 \mathrm{~cm}^{-1}$ peak and time was obtained according to the same procedure. The results of the noncomplementary miRNA and single-base mismatch miRNA hybridizations are shown in Figure 4(e) which were represented by black squares and red circles, respectively. It could be observed that the peak at $1334 \mathrm{~cm}^{-1}$ changed slightly when the SERS platform was employed to test the noncomplementary miRNA after more than 50 minutes. Similarly, compared to the noncomplementary miRNA, the quenched SERS intensity of the single-base mismatch miRNA was stronger. However, it was obviously lower than that of the complementary miRNA. The complementary detection of miR-196a indicated a monotonic decay at the peak of $1334 \mathrm{~cm}^{-1}$, and a relatively stable peak was observed at a time of more than 110 minutes. Figure 4(f) indicates that the stable SERS spectra of the platform before and after miRNA hybridization with complementary, noncomplementary, and single-base mismatch for direct comparison. It was clear that characteristic peaks at $1640 \mathrm{~cm}^{-1}$ and $1184 \mathrm{~cm}^{-1}$ were different although these spectra showed similar SERS peaks. The results showed that the SERS platform could effectively and specifically identify the target. Compared with the complementary miR$196 \mathrm{a}$, even the single nucleotide miRNA could not cause the expected changes of SERS intensity.

\subsubsection{Uniformity and Reproducibility of SERS Platform.} The uniformity of the SERS platform was an important parameter. The homogeneity of the platform was estimated by testing SERS signals intensity on six random spots of the SERS platform after hybridization with 100 pM miR-196a (Figure $4(\mathrm{~g})$ ). The little difference at $1334 \mathrm{~cm}^{-1}$ indicated that the SERS platform had good uniformity (Figure 4(h)). We further studied the reproducibility of the SERS platform. Three platforms were prepared at different times and were utilized to detect the $100 \mathrm{pM}$ miR-196a (Figure 4(i)). The average SERS spectrum was obtained by scanning different points 10 times by SERS for each SERS platform as well as comparing Raman intensities at $1334 \mathrm{~cm}^{-1}$. As shown in Figure $4(\mathrm{j})$, the deviation of the peak intensity at $1334 \mathrm{~cm}^{-1}$ was $6.6 \%$. Thus, the SERS platform was uniform and reproducible which could be used as a candidate material for miRNA detection. In order to better assess the specificity and the sensibility of the SERS platform, SERS was taken to detect miR-196a in healthy people matched for gender and sex. As shown in Figure S2 (supporting information), eight healthy men and eight healthy women were randomly selected from 30 healthy people and their serums were detected by SERS. The deviation of the male group (1-8) was $8.76 \%$, that of the female group (9-16) was $4.65 \%$, and the average deviation between male and female groups was $1.52 \%$. Therefore, the platform possessed good specificity and sensibility, which demonstrated the high precision in detection for miR-196a in clinical samples.

3.5. Detection of miR-196a in PBS and Serum. As shown in Figure 5(a), before and after the hairpin DNA hybridization, the SERS spectra of the 5-FAM were obtained at various concentrations of miR-196a from $1 \mathrm{fM}$ to $100 \mathrm{pM}(1 \mathrm{fM}$, $10 \mathrm{fM}, 100 \mathrm{fM}, 1 \mathrm{pM}, 10 \mathrm{pM}$, and $100 \mathrm{pM}$ ) in PBS buffer. It was obvious that the SERS intensities gradually decreased with increasing hybridized miR-196a concentration in comparison with the SERS spectrum before hybridization (black curve, designated as "original”). The SERS intensity of the peak at $1334 \mathrm{~cm}^{-1}$ was set as a function of miR-196a concentration $\left(C_{\text {miR-196a }}\right)$ in Figure 5(b) which was shown as a semilog scale, where $\Delta I=I_{0}-I, I$ was the intensity after hybridization while $I_{0}$ was the original SERS intensity. $\Delta I$ nearly followed a linear relationship with $\log \left[\mathrm{C}_{\mathrm{miR}-196 \mathrm{a}}\right]$, and the regression equation could be expressed as $y=1124 \log$ $\left[\mathrm{C}_{\mathrm{miR}-196 \mathrm{a}}\right]+18001$ and $R^{2}$ was 0.9705 . The LOD was calculated to be 96.58 aM.

The application of the SERS platform was further studied by detecting the concentration of miR-196a in serum. By mixing miRNA with the serum, miR-196a solutions were obtained with final concentrations ( $1 \mathrm{fM}-100 \mathrm{pM})$. The Raman intensities of the peaks at $1334 \mathrm{~cm}^{-1}$ increased proportionally with the concentration of the miR-196a in serum as shown in Figure 5(c). Figure 5(d) shows that the calibration curve of miR-196a exhibited a linear relationship from $1 \mathrm{fM}$ to $100 \mathrm{pM}$. The regression equation and correlation coefficient were $y=1195.2 \log \left[\mathrm{C}_{\mathrm{miR}-196 \mathrm{a}}\right]+18987$ and 0.9864 , and the calculated lowest detectable concentration was $130 \mathrm{aM}$ aM for miR-196a in serum. It exhibited superior performance to the previously reported SERS miRNA platform, and the signal amplification is shown in Table 3. The results indicate that this SERS platform is a very useful analytical tool for the rapid and highly sensitive detection of miRNA biomarkers in serum.

3.6. Differentiation of miR-196a Expression in Serum of Lung Cancer Patients and Healthy People by SERS and RT-PCR. To validate the applicability of the SERS platform in distinguishing miR-196a expression in serum of lung cancer patients and healthy people, serum was directly dropped onto the SERS platform for the detection of miR-196a. Their SERS spectra were obtained by averaging ten different locations on the SERS platform. Figure 6(a) shows the corresponding SERS spectra of the average SERS spectra of miR-196a concentration in serum collected from pure SERS substrate, incubated with serum of lung cancer patients and healthy people. The blank groups represented pure SERS substrate before incubation with the target miRNA, which exhibited a higher SERS intensity than that of lung cancer patients and healthy people because the miR-196a existed in 


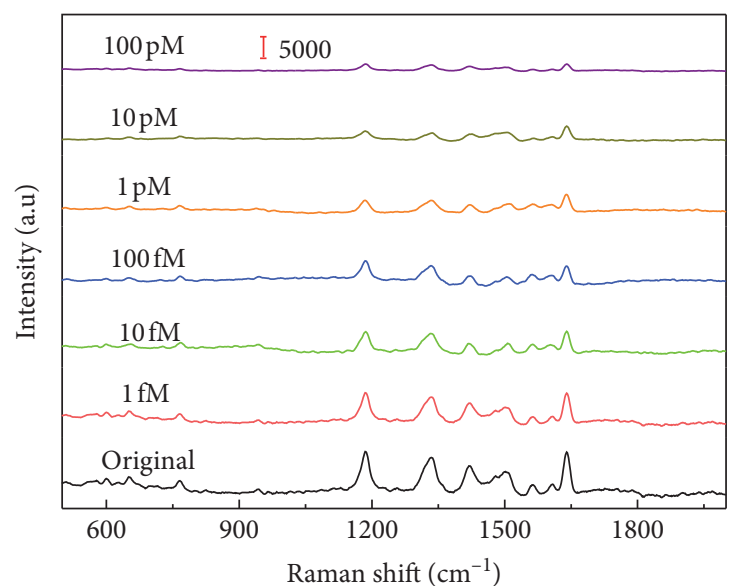

(a)

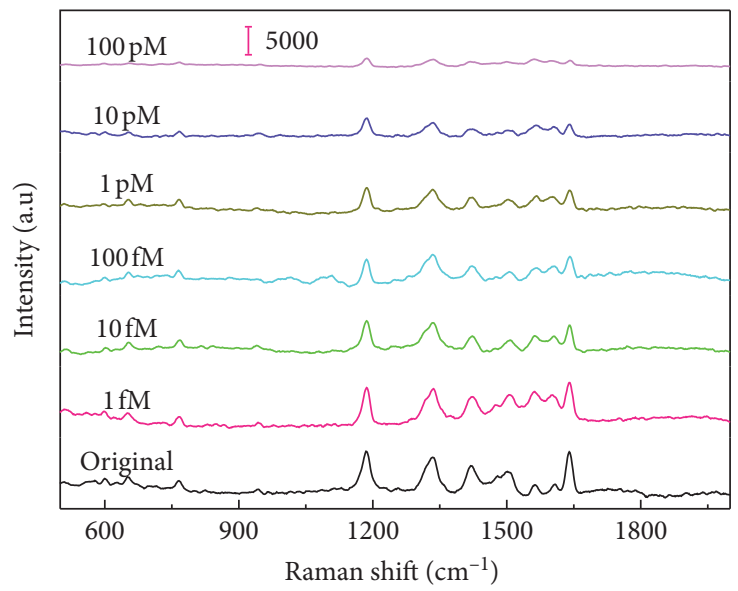

(c)

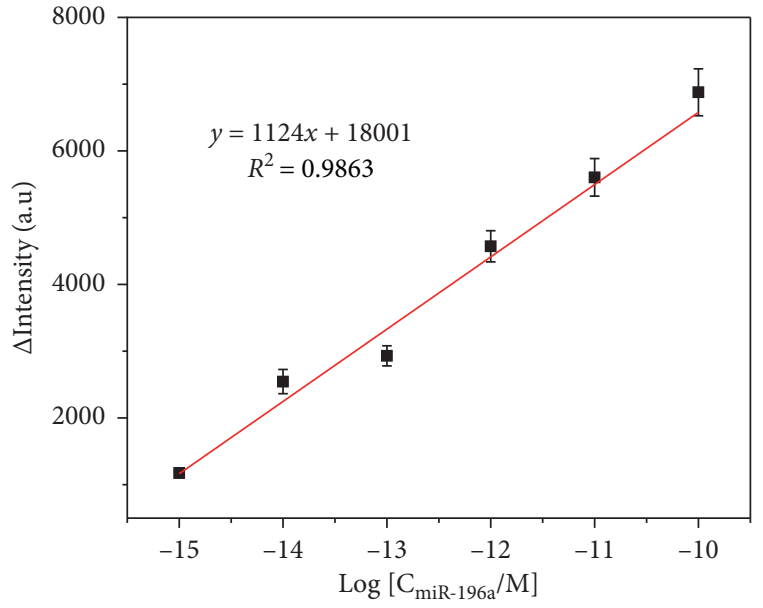

(b)

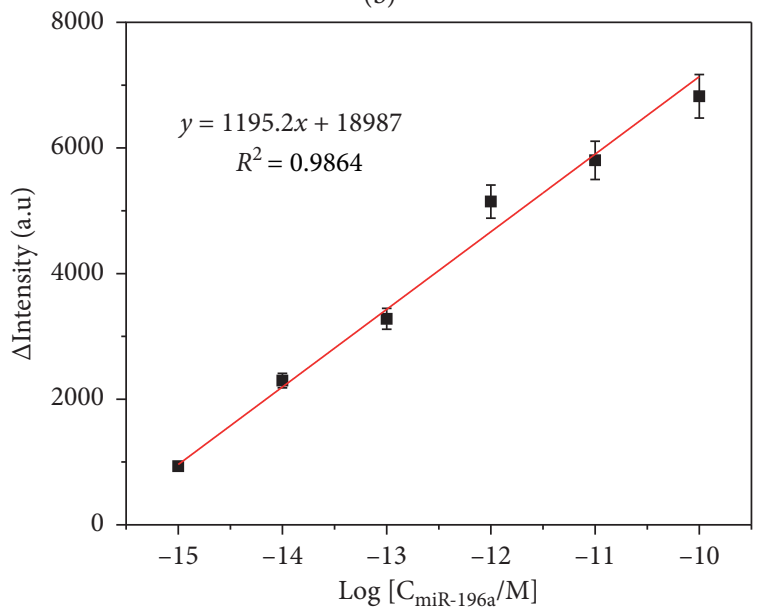

(d)

FIGURE 5: (a) SERS spectra of 5-FAM obtained before and after hairpin DNA hybridization at various concentrations of miR-196a in PBS. (b) The calibration curve of SERS intensities of $\Delta I_{1334}$ versus the miRNA concentration in PBS buffer. (c) Corresponding SERS spectra of 5FAM collected before and after hairpin DNA hybridization by various concentrations of miR-196a in serum. (d) The calibration curve of SERS intensities of $\Delta I_{1334}$ versus the miRNA concentration in serum. The red scale bars represent 5000 a.u.

TABLE 3: Comparison of the AgNW@AuNPs assembly-based SERS platform to other SERS miRNA probes with signal amplification.

\begin{tabular}{lccc}
\hline Detection target & LOD $(\mathrm{fM})$ & Linear ranges & \\
\hline miR-203 & 0.15 & $10^{-15} \sim 10^{-12} \mathrm{M}$ & Reference \\
miR-141 & 0.17 & $10^{-15} \sim 10^{-7} \mathrm{M}$ & {$[39]$} \\
miR-203 & 6.3 & $10^{-15} \sim 10^{-8} \mathrm{M}$ & {$[40]$} \\
miR-155 & 0.11 & $10^{-15} \sim 10^{-10} \mathrm{M}$ & {$[41]$} \\
Let-7b & 0.3 & $10^{-15} \sim 10^{-9} \mathrm{M}$ & {$[42]$} \\
miR-155 & 83 & $10^{-15} \sim 10^{-10} \mathrm{M}$ & {$[43]$} \\
miR-196a & 96.58 & $10^{-15} \sim 10^{-10} \mathrm{M}$ & {$[44]$} \\
\hline
\end{tabular}

serum which resulted in the hybridized hairpin DNA and the SERS signal weakening. The intensity of the SERS signals from healthy people serum was higher than lung cancer patients for the cause that the expression of miR-196a was downregulated in normal human serum compared with lung cancer serum [45]. The Raman intensities at $1334 \mathrm{~cm}^{-1}$ were entered into the calibration curve equation (Figure $5(\mathrm{~d})$ ) to determine the miR-196a concentrations. The concentrations of the miR-196a in the serum of lung cancer patients and healthy people were 20.19 and $4.98 \mathrm{fM}$, respectively. Meanwhile, the SERS assay for the miR-196a expression in the serum of lung cancer patients was consistent with the RT-PCR results (Figure 6(b)). Therefore, the prepared SERS platform shows the potential for the rapid and accurate detection of miRNA expression in serum of cancer patients. 


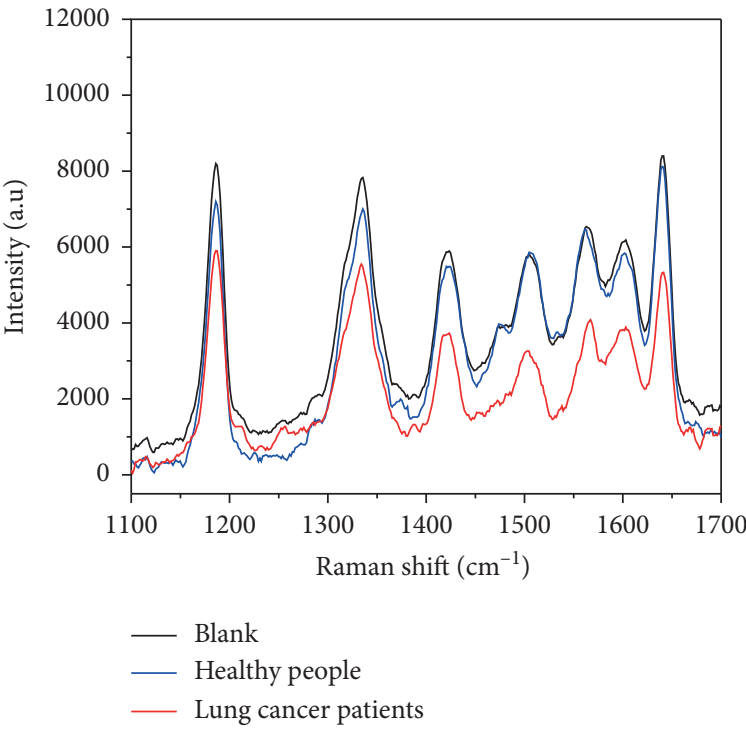

(a)

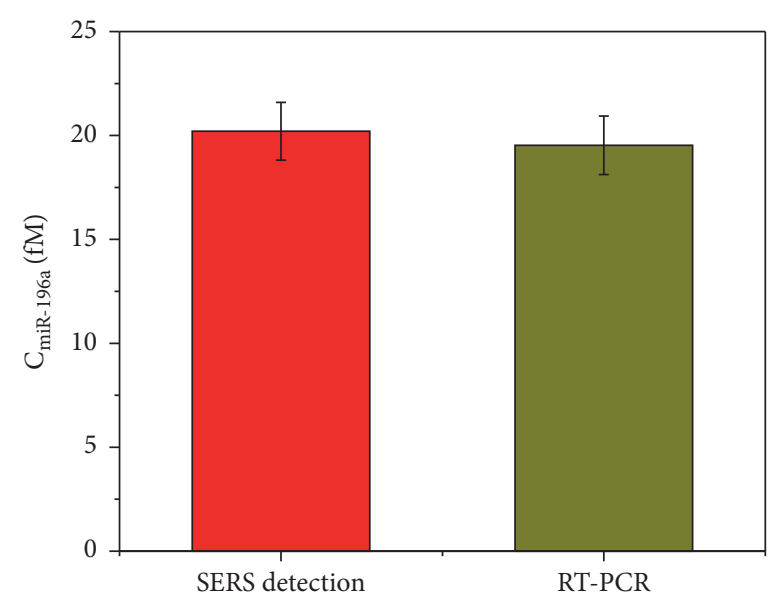

(b)

FIGURE 6: (a) SERS spectra of miR-196a expression in serum of lung cancer patients and healthy people. (b) The expression of miR-196a in serum of lung cancer patients detected by SERS platform and RT-PCR.

\section{Conclusions}

In summary, a rapid, accurate, and sensitive SERS platform was developed for the detection of the miRNA biomarkers in the serum of lung cancer patients. FDTD had proved that a strong SERS signal could be produced by hot spots formation in the gaps of AgNW@AuNPs.AgNW@AuNPs were synthesized and assembled onto the surface of HDSA-treated filter paper to prepare SERS substrates. The SERS platform was prepared by immobilizing hairpin-shaped DNA molecules related to miR196a on an AgNW@AuNP substrate. The results showed that the SERS platform had the advantages of high sensitivity, good specificity, perfect uniformity, and favorable reproducibility. SERS platform could achieve a linear at the range of $1 \mathrm{fM}$ to $100 \mathrm{pM}$ and the LODs were $96.58 \mathrm{aM}$ in the PBS and $130 \mathrm{aM}$ in serum with SERS signal measured after hybridization with the target miR-196a. Finally, the SERS platform was further employed for the detection of miR-196a expression in the serum of lung cancer patients and healthy people. The expression of miR-196a in the serum of lung cancer patients was much higher than that of healthy people. The SERS results were consistent with the RT-qPCR analysis of the miR-196a concentration detection. The hairpin DNA-functionalized SERS platform could be used for the detection of miRNA in serum with the potential for the diagnosis of cancer.

\section{Data Availability}

The data used to support the findings of this study are included within the article.

\section{Conflicts of Interest}

The authors declare no conflicts of interest.

\section{Acknowledgments}

The authors thank LetPub (http://www.letpub.com) for its linguistic assistance during the preparation of this manuscript. The authors gratefully acknowledge support from the National Natural Science Foundation of China (no. 81701825), the Natural Science Foundation of the Jiangsu Higher Education Institutions of China (no. 17KJB416012), and the Postdoctoral Science Foundation in Jiangsu Province (no. 1701141C).

\section{Supplementary Materials}

Figure S1: SERS mapping for NBA on ordinary filter paperbased AgNW@AuNPs substrate. Figure S2: (A) SERS spectra of the serum of healthy men and healthy women $\left(1334 \mathrm{~cm}^{-1}\right)$; (B) the peak intensity histogram of the SERS spectra in (A). (Supplementary Materials)

\section{References}

[1] L. S. Chan, P. Y. K. Yue, Y. Y. Wong, and R. N. S. Wong, "MicroRNA-15b contributes to ginsenoside-Rg1-induced angiogenesis through increased expression of VEGFR-2," Biochemical Pharmacology, vol. 86, no. 3, pp. 392-400, 2013.

[2] B. P. Lewis, C. B. Burge, and D. P. Bartel, "Conserved seed pairing, often flanked by adenosines, indicates that thousands of human genes are microRNA targets," Cell, vol. 120, no. 14, pp. 15-20, 2005.

[3] R. Schickel, B. Boyerinas, S.-M. Park, and M. E. Peter, "MicroRNAs: key players in the immune system, differentiation, tumorigenesis and cell death," Oncogene, vol. 27, no. 45, pp. 5959-5974, 2008.

[4] T.-H. Chen, C. Lee, C.-T. Chiu et al., "Circulating microRNA$196 \mathrm{a}$ is an early gastric cancer biomarker," Oncotarget, vol. 9, no. 12, pp. 10317-10323, 2018. 
[5] H. Y. Kim, J.-H. Yoon, H.-S. Lee et al., "MicroRNA-196A-2 polymorphisms and hepatocellular carcinoma in patients with chronic hepatitis B," Journal of Medical Virology, vol. 86, no. 3, pp. 446-453, 2014.

[6] C. Z. Liu, J. F. Lin, L. Q. Li et al., "HPV16 early gene E5 specifically reduces miRNA-196a in cervical cancer cells," Science Report, vol. 5, p. 7653, 2015.

[7] T. Li, L. Niu, L. Wu et al., "A functional polymorphism in microRNA-196a2 is associated with increased susceptibility to non-Hodgkin lymphoma," Tumor Biology, vol. 36, no. 5, pp. 3279-3284, 2015.

[8] M. G. Albrecht and J. A. Creighton, "Anomalously intense Raman spectra of pyridine at a silver electrode," Journal of the American Chemical Society, vol. 99, no. 15, pp. 5215-5217, 1977.

[9] J. A. Dieringer, R. B. Lettan, K. A. Scheidt, and R. P. Van Duyne, "A frequency domain existence proof of single-molecule surface-enhanced Raman spectroscopy," Journal of the American Chemical Society, vol. 129, no. 51, pp. 16249-16256, 2007.

[10] K. A. Willets, "Surface-enhanced Raman scattering (SERS) for probing internal cellular structure and dynamics," Analytical and Bioanalytical Chemistry, vol. 394, no. 1, pp. 85-94, 2009.

[11] J.-Y. Huang, C. Zong, L.-J. Xu, Y. Cui, and B. Ren, "Clean and modified substrates for direct detection of living cells by surface-enhanced Raman spectroscopy," Chemical Communications, vol. 47, no. 20, pp. 5738-5040, 2011.

[12] D. Lu, J. Xia, Z. Deng, and X. Cao, "Detection of squamous cell carcinoma antigen in cervical cancer by surface-enhanced Raman scattering-based immunoassay," Analytical Methods, vol. 11, no. 21, pp. 2809-2818, 2019.

[13] H. Zhou, D. Yang, N. P. Ivleva, N. E. Mircescu, R. Niessner, and C. Haisch, "SERS detection of bacteria in water by in situ coating with Ag nanoparticles," Analytical Chemistry, vol. 86, no. 3, pp. 1525-1533, 2014.

[14] J. Zhang, Y. Dong, W. Zhu et al., "Ultrasensitive detection of circulating tumor DNA of lung cancer via an enzymatically amplified SERS-based frequency shift assay," ACS Applied Materials \& Interfaces, vol. 11, no. 20, pp. 18145-18152, 2019.

[15] W.-F. Zhu, L.-X. Cheng, M. Li et al., "Frequency shift Ramanbased sensing of serum MicroRNAs for early diagnosis and discrimination of primary liver cancers," Analytical Chemistry, vol. 90, no. 17, pp. 10144-10151, 2018.

[16] B. Guven, F. C. Dudak, I. H. Boyaci, U. Tamer, and M. Ozsoz, "SERS-based direct and sandwich assay methods for mir-21 detection," The Analyst, vol. 139, no. 5, pp. 1141-1147, 2014.

[17] Y. Pang, C. Wang, J. Wang, Z. Sun, R. Xiao, and S. Wang, " $\mathrm{Fe}_{3} \mathrm{O}_{4} @ \mathrm{Ag}$ magnetic nanoparticles for microRNA capture and duplex-specific nuclease signal amplification based SERS detection in cancer cells," Biosensors and Bioelectronics, vol. 79, no. 15 , pp. 574-580, 2016.

[18] K. A. Willets and R. P. Van Duyne, "Localized surface plasmon resonance spectroscopy and sensing," Annual Review of Physical Chemistry, vol. 58, no. 1, pp. 267-297, 2007.

[19] H.-L. Jiang, T. Akita, T. Ishida, M. Haruta, and Q. Xu, "Synergistic catalysis of Au@Ag Core-Shell nanoparticles stabilized on Metal-Organic framework," Journal of the American Chemical Society, vol. 133, no. 5, pp. 1304-1306, 2011.

[20] P. Li, B. Ma, L. Yang, and J. Liu, "Hybrid single nanoreactor for in situ SERS monitoring of plasmon-driven and small Au nanoparticles catalyzed reactions," Chemical Communications, vol. 51, no. 57, pp. 11394-11397, 2015.
[21] J. W. Benjamin, H. I. Sang, Z. Y. Li, M. Joeseph, S. Andrew, and Y. Xia, "Maneuvering the surface plasmon resonance of silver nanostructures through shape-controlled synthesis," Journal of Physical Chemistry B, vol. 110, no. 32, pp. 1566615675, 2006.

[22] Y. Sun and Y. Xia, "Gold and silver nanoparticles: a class of chromophores with colors tunable in the range from 400 to 750 nm," The Analyst, vol. 128, no. 6, pp. 686-691, 2003.

[23] W. K. Mark, K. G. Nathaniel, B. Rizia, H. Feng, N. Peter, and J. H. Naomi, "Nanoparticle-mediated coupling of light into a nanowire," Nano Letters, vol. 7, no. 8, pp. 2346-2350, 2007.

[24] W. Kim, J.-C. Lee, G.-J. Lee, H.-K. Park, A. Lee, and S. Choi, "Low-cost label-free biosensing bimetallic cellulose strip with SILAR-synthesized silver core-gold shell nanoparticle structures," Analytical Chemistry, vol. 89, no. 12, pp. 6448-6454, 2017.

[25] W. Zhang, B. Li, L. Chen et al., "Brushing, a simple way to fabricate SERS active paper substrates," Analytical Methods, vol. 6, no. 7, pp. 2066-2071, 2014.

[26] B. Li, W. Zhang, L. Chen, and B. Lin, "A fast and low-cost spray method for prototyping and depositing surface-enhanced Raman scattering arrays on microfluidic paper based device," Electrophoresis, vol. 34, no. 15, pp. 2162-2168, 2013.

[27] R. Wang, Y. Xu, R. Wang et al., "A microfluidic chip based on an ITO support modified with Ag-Au nanocomposites for SERS based determination of melamine," Microchimica Acta, vol. 184, no. 1, pp. 279-287, 2016.

[28] X. Fu, Z. Cheng, J. Yu, P. Choo, L. Chen, and J. Choo, “A SERS-based lateral flow assay biosensor for highly sensitive detection of HIV-1 DNA," Biosensors and Bioelectronics, vol. 78, pp. 530-537, 2016.

[29] W. W. Yu and I. M. White, "Inkjet-printed paper-based SERS dipsticks and swabs for trace chemical detection," The Analyst, vol. 138, no. 4, pp. 1020-1025, 2013.

[30] R. Tantra, R. J. C. Brown, M. J. T. Milton, and D. Gohil, “A practical method to fabricate gold substrates for surface-enhanced Raman spectroscopy," Applied Spectroscopy, vol. 62, no. 9, pp. 992-1000, 2008.

[31] R. Zhang, B.-B. Xu, X.-Q. Liu et al., "Highly efficient SERS test strips," Chemical Communications, vol. 48, no. 47, pp. 5913-5915, 2012.

[32] L. Burr, I. Schubert, W. Sigle, C. Trautmann, and M. E. ToimilMolares, "Surface enrichment in Au-Ag alloy nanowires and investigation of the dealloying process," The Journal of Physical Chemistry C, vol. 119, no. 36, pp. 20949-20956, 2015.

[33] H. T. Ngo, H.-N. Wang, A. M. Fales, and T. Vo-Dinh, "Labelfree DNA biosensor based on SERS molecular sentinel on nanowave chip," Analytical Chemistry, vol. 85, no. 13, pp. 6378-6383, 2013.

[34] J. Dong, Y. Li, M. Zhang, Z. Li, T. Yan, and W. Qian, "Ultrasensitive surface-enhanced Raman scattering detection of alkaline phosphatase," Analytical Methods, vol. 6, no. 22, pp. 9168-9172, 2014.

[35] X. T. Wang, W. S. Shi, G. W. She, L. X. Mu, and S. T. Lee, "High-performance surface-enhanced Raman scattering sensors based on Ag nanoparticles-coated Si nanowire arrays for quantitative detection of pesticides," Applied Physics Letters, vol. 96, Article ID 053104, 2010.

[36] L. L. Wang, A. Roitberg, C. Meuse, and A. K. Gaigalas, "Raman and FTIR spectroscopies of fluorescein in solutions," Spectrochimica Acta Part A, vol. 57, no. 9, pp. 1781-1791, 2011.

[37] K. K. Strelau, A. Brinker, C. Schnee, K. Weber, R. Möller, and J. Popp, "Detection of PCR products amplified from DNA of epizootic pathogens using magnetic nanoparticles and SERS," 
Journal of Raman Spectroscopy, vol. 42, no. 3, pp. 243-250, 2011.

[38] S. J. Ye, Y. Y. Guo, J. Xiao, and S. S. Zhang, "A sensitive SERS assay of L-histidine via a DNAzyme-activated target recycling cascade amplification strategy," Chemical Communications, vol. 49, no. 3, pp. 3643-3645, 2013.

[39] S. Ye, Y. Wu, X. Zhai, and B. Tang, "Asymmetric signal amplification for simultaneous SERS detection of multiple cancer markers with significantly different levels," Analytical Chemistry, vol. 87, no. 16, pp. 8242-8249, 2015.

[40] X. Li, S. Ye, and X. Luo, "Sensitive SERS detection of miRNA via enzyme-free DNA machine signal amplification," Chemical Communications, vol. 52, no. 67, pp. 10269-10272, 2016.

[41] H. Zhang, Y. Liu, J. Gao, and J. Zhen, "A sensitive SERS detection of miRNA using a label-free multifunctional probe," Chemical Communications, vol. 51, no. 94, pp. 16836-16839, 2015.

[42] Y. He, X. Yang, R. Yuan, and Y. Chai, “"Off” to "on" surface-enhanced Raman spectroscopy platform with padlock probe-based exponential rolling circle amplification for ultrasensitive detection of MicroRNA 155," Analytical Chemistry, vol. 89, no. 5, pp. 2866-2872, 2017.

[43] Y. Pang, C. Wang, L. Lu, C. Wang, Z. Sun, and R. Xiao, "DualSERS biosensor for one-step detection of microRNAs in exosome and residual plasma of blood samples for diagnosing pancreatic cancer," Biosensors and Bioelectronics, vol. 130, no. 1, pp. 204-213, 2019.

[44] Y. He, X. Yang, R. Yuan, and Y. Chai, "Switchable targetresponsive 3D DNA hydrogels as a signal amplification strategy combining with SERS technique for ultrasensitive detection of miRNA 155," Analytical Chemistry, vol. 89, no. 16, pp. 8538-8544, 2017.

[45] I. Guerriero, D. D. Angelo, P. Pallante et al., "Analysis of miRNA profiles identified miR-196a as a crucial mediator of aberrant PI3K/AKT signaling in lung cancer cells," Oncotarget, vol. 8, no. 12, pp. 9172-9191, 2017. 